\title{
Capital Intelectual, Gestión del Conocimiento en la Interacción Gobierno y Gestión de las Tecnologías de la Información desde Perspectiva COBIT 5
}

\section{Intellectual capital, knowledge management in the interaction government and management of information technologies from COBIT 5 perspective}

Percy Edwin De la Cruz Vélez de Villa ${ }^{1}$

Universidad Nacional Mayor de San Marcos, Perú

Recibido: 10-06-2017

Aceptado: 07-12 -2017

\section{Cita Recomendada}

De la Cruz, P. (2017) Capital Intelectual, Gestión del Conocimiento en la Interacción Gobierno y Gestión de las Tecnologías de la Información desde Perspectiva COBIT 5. Hamut'ay, 4 (2), 30-44. Recuperado de: http://dx.doi.org/10.21503/hamu.v4i2.1470

\section{RESUMEN}

Este artículo hace una reflexión sobre el Capital Intelectual y la Gestión del Conocimiento desde una propuesta de perspectiva COBIT 5 (Control Objectives for Information and Related Technology), exponiendo la importancia para alcanzar objetivos estratégicos y obtener un nivel de calidad idóneo, a través de la eficiencia, eficacia y efectividad en todo el ámbito de procesos y procedimientos orientados al Desarrollo Organizacional, por lo que el objetivo es presentar estos conceptos a través de la investigación documental analizada en base de datos, Google académico, catálogos on-line y repositorios. La propuesta de perspectiva COBIT 5, se describe en su Principio 4 "Habilitar un enfoque holístico". En la explicación se detalla cada habilitador y su interacción con el Capital Intelectual y Gestión del Conocimiento, en la que se observa la viabilidad para lograr los objetivos y metas institucionales asegurando y cerrando la brecha entre Gestión de Tecnologías de la Información y los objetivos del negocio. Se concluye del análisis que la participación de estos dos conceptos en todos los procesos es muy activa e importante, por lo que se propone su definición explícita en los modelos de Gobierno.

Palabras Clave: Gobierno de la Tecnología de Información, Gestión de la Tecnología de Información, Capital Intelectual, Gestión del Conocimiento, ISO/IEC 38500: 2015, COBIT 5.

\section{Abstract}

This article provides a reflection on Intellectual Capital and Knowledge Management out of a COBIT 5 framework proposal (Control Objectives for Information and Related Technology), stating the importance to achieve strategic objectives and to obtain an ideal quality level, through efficiency, efficacy and effectiveness in the whole area of processes and procedures oriented to the Orga-

1 Magister en Computación e Informática, especialista en Tecnologías de la Información, profesor principal de la Universidad Nacional Mayor de San Marcos. Email: Pdelacruzv@unmsm.edu.pe 
nizational Development; thus, the objective is to present these concepts through a documentary investigation analyzed in data bases, Google Scholar, on-line catalogs and repositories. The COBIT 5 framework proposal is described in its Principle 4 "Enabling a holistic approach". In the explanation, each enabler and its interaction with the Intellectual Capital and Knowledge Management is detailed, in which the viability to achieve the institutional objectives and goals is observed, ensuring and closing the gap between Information Technology Management and business objectives. From the analysis, it is concluded that the participation of these two concepts in all the processes is very active and important, so its explicit definition is proposed in the Governance models.

Keywords: Information Technology Governance, Information Technology Management, Intellectual Capital, Knowledge Management, ISO/IEC 38500: 2015, COBIT 5.

\section{INTRODUCCIÓN}

En la sociedad actual el conocimiento asociado al capital humano como activo intangible es un factor clave de éxito para hacer a las organizaciones más competitivas, la gestión de ese conocimiento esta basado en la evolución de la Gestión de la Información hacía la generación de Valor; este es el punto motivacional para la propuesta entendiendo que el objetivo de cualquier institución se enmarca en la supervivencia, rentabilidad y crecimiento.

Según Steward (1998) la Gestión del Conocimiento (GC) es el conjunto de procesos que hacen que el Capital Intelectual (CI) de la empresa crezca. El CI está ganando importancia día a día como un enfoque para la medición de los intangibles, especialmente en el contexto de crecimiento de la economía basada en el conocimiento, sobre el cual Akpinar \& Akdemir (1999) precisan que la riqueza económica en la actualidad se basa en el conocimiento y ya no en el proceso de producción.

Respecto a la tecnología, el objetivo principal del Gobierno de la Tecnología de Información (GTI) es conseguir la alineación entre la estrategia del negocio y la estrategia de las Tecnologías de la Información (TI), cerrar esta brecha es fundamental para que la Gestión de las Tecnologías de la Información cumpla su función primordial de generación de valor para los grupos de interés, minimizando los riesgos y optimizando los recursos. Rodríguez, Aguilar y Raudales (2017) manifiestan que, a fin de ordenar, es plausible controlar la GC mediante implementación en la empresa de certificaciones, haciendo uso de estándares como COBIT 5 (2012) o estándares de la Organización Internacional de Normalización (por sus siglas en inglés-ISO). En la versión 5, el COBIT diferencia con más detalle el Gobierno de la Gestión de la TI. Siguiendo este marco de referencia, las empresas aseguran el logro de sus objetivos; sin embargo, en el COBIT 5 y en la normatividad actual de GTI no está explícitamente definida la participación de CI y la GC. Se propone que sólo entendiendo y considerando desde su concepción en el marco, así como en la norma ISO/IEC 38500: 2015 centrada en el gobierno y derivada en la gestión de TI, se consolida esta, para que la ejecución sea exitosa.

El objetivo es presentar un estudio preliminar de reflexión sobre el concepto y la relación del CI, GC en el Gobierno y la Gestión de la TI, bajo la perspectiva del COBIT 5 mediante sus siete habilitadores. Para lo cual se ha considerado como objetivos específicos los siguientes:

1. Definir conceptos como: GC, CI, GTI, Gestión de la TI, se aborda el COBIT 5 tangencialmente para focalizar el principio 4 y los 7 habilitadores, detallando como en su accionar están involucrados las personas, la GC y el CI.

2. Tomar como base el COBIT 5, ya que provee a las empresas un marco de trabajo integral que ayuda a alcanzar sus objetivos para el gobierno y la gestión de TI.

3. Determinar cómo influye la GC en el desarrollo organizacional, lo cual se muestra en la tabla 1. 
4. Analizar y sintetizar la revisión bibliográfica relacionada a la GC vinculada al CI, asimismo identificar qué aporte o método utilizan, lo cual se describe en la tabla 2.

5. Analizar los siete habilitadores del COBIT 5 y se identifica su relación con GC, CI con Capital Humano $(\mathrm{CH})$, Capital Estructural (CE) y Capital Relacional (CR), como se detalla en la tabla 3.

6. Identificar, ubicar y mostrar como la GC, CI están en cada tarea, actividad y proceso, lo cual se presenta en las conclusiones.

\section{Método}

Este trabajo es una investigación de revisión documental, en la cual se explora la literatura referente al CI, GC, los marcos de gobierno de TI como: la ISO / IEC 38500: 2015 (Calder-Moir, 2013). Así también el COBIT 5 relacionando al Gobierno y Gestión de la TI.

El método utilizado fue:

a. Ubicar el tema de análisis (taxonomía) en: Association for Computing Machinery (ACM)

Applied computing

Enterprise computing

IT governance

Institute of Electrical and Electronics Engineers (IEEE)

M.7.0 Governance

M.7.0.c Norms and regulations

M.7.0.d Policies

M.9.0 Architecture

M.9.1 Bridging business and IT

M.9.1.1. IT architectures

M.9.1.2. IT governance

b. La selección del material bibliográfico y documental se apoyó en Google Académico para COBIT 5 y otros fundamentos teóricos. Para la GC, CI, así como para el GTI, en repositorios, bibliotecas digitales y bases de datos como: Scielo, ACM, PROQUEST, ELSE-

\section{VIER, ScienceDirect.}

c. Criterios de inclusión. Se consideraron publicaciones de artículos, libros y otro material de lectura de los últimos seis años en el área de la temática mencionada líneas anteriores; asimismo, en la búsqueda de la información se utilizaron palabras claves como capital intelectual, gestión del conocimiento, gestión de tecnologías de la información, COBIT 5, Knowledge management, government, Intellectual capital and management of information technologies.

d. De toda la literatura presentada, son nueve artículos los que sirvieron de base para el trabajo y construcción de las tablas correspondientes, los cuales fueron complementados con libros digitales, físicos y artículos de revistas indexadas como se detalla en las referencias bibliográficas, los cuales fundamentaron el marco teórico del estudio.

En la tabla 1, se observa la importancia que tiene la GC en las organizaciones, en la tabla 2 se identifican los artículos seleccionados y su relación con el CI y/o GC; asimismo se describe el método y aporte. En la tabla 3, con base al COBIT 5, se relaciona los habilitadores (7) con la GC y el CI; en este análisis por cada habilitador se precisa cómo o dónde está la participación de los conceptos: $\mathrm{CI}$ y GC.

La intención es mostrar que los conceptos CI y GC están presentes y cumplen un rol importantísimo y, los marcos de referencia de Gobierno y Gestión de la TI, no manifiestan explícitamente sus aportes.

Por otro lado, de los nueve artículos como base del estudio el $88 \%$ cumplen estrictamente los criterios de inclusión. Debemos precisar que el artículo de Vidovic (2010) no ha sido sometido a revisión o aprobación formal; pero lo han citado ya artículos que están en PROQUEST así cómo tesis de maestría entre otros, motivo por el cual se considera válido como fuente de referencia en este estudio. 


\section{Capital intelectual (CI)}

También se conoce como el nuevo activo invisible y la definición más ampliamente utilizado de CI es "el conocimiento que da valor a una organización" o como lo refiere Villegas, Hernández y Salazar (2015 p. 184) el conocimiento es una fuente primordial que propicia la creación y generación de ventaja competitiva y riqueza en las organizaciones. A partir de esta definición se concluye que la GC genera CI. Por lo tanto, la supervivencia y el éxito competitivo de las empresas dependerán mucho de la gestión estratégica de su $\mathrm{CI}$ en comparación con los recursos financieros. En la economía basada en el conocimiento, para ser una nación desarrollada y para mantener el estado desarrollado, el tener un capital humano de alta calidad es una prioridad. Además, para aumentar la competitividad de una organización, la mano de obra debe ser más eficiente, adaptable y competente. Por este motivo muchas organizaciones, e inclusive países, han entendido de la urgencia de su aplicación y es un punto de la agenda principal en el marco del Noveno Plan de Malasia y el nuevo modelo económico de 2010.

El CI es tan importante como la inversión de capital para las empresas. Por lo tanto, los gobiernos de los países deberían equilibrar estas inversiones, más aún los países en vías de desarrollo (Chen, Cheng \& Hwang, 2005). De acuerdo con Bontis \& Richardson (2000), hay tres elementos principales del CI que son: el Capital Humano $(\mathrm{CH})$, Capital Estructural (CE) y el Capital Cliente también llamado Capital Relacional (CR), y es a través de esta combinación que se crea valor para la organización, el análisis correspondiente al CI es para determinar su riqueza intelectual.

Las empresas que poseen todos los componentes del Capital Intelectual, están en mejores condiciones para competir en el negocio (Amrizah \& Rashidah, 2013).

Según Wiig (1997) el conocimiento y el CI son conceptos relacionados, pero, en su gestión, sus objetivos y alcances son diferentes; ambos son los activos más importantes de una organización. La gestión del CI se concentra básicamente en renovar y maximizar el valor de los activos intelectuales de la organización, la GC se concentra en faci- litar y gestionar las actividades relacionadas con el conocimiento (creación, captura, transformación y uso); su función consiste en planear, implementar, operar y monitorear todas las actividades y programas relacionados con el conocimiento que se requiere para una gestión eficaz del CI (Wiig, 1997).

El Capital Humano $(\mathrm{CH})$ como elemento del capital intelectual, según Fitz - Enz (2009) menciona que este es una combinación de tres factores: a) el carácter o la naturaleza que se lleva al trabajo; por ejemplo, inteligencia, energía, actitud positiva, fiabilidad y compromiso, b) las capacidades de la persona para aprender, tales como la inteligencia, la imaginación, la creatividad y c) la motivación del talento para compartir información o conocimiento, el espíritu de equipo y la orientación de meta.

Como otro de los elementos del capital intelectual el Capital Estructural (CE), que es la infraestructura que apoya a los empleados para crear un rendimiento óptimo, incluyendo la capacidad de la organización para llegar al mercado, hardware, software, bases de datos, estructura organizativa, patentes, marca registrada y toda capacidad de las organizaciones para apoyar la productividad de los empleados (Bontis, et al., 2000). El concepto de la existencia de CE habilita la creación de CI y de enlace-procesamiento de los recursos humanos. Según Gogana et al., (2014), son 15 conceptos clave los que definen el CE, creados por las personas en su trabajo cotidiano en la organización (véase figura 1). Estas palabras deben capitalizarse por medio de la GC. Permitiendo y asegurando el desarrollo de las organizaciones.

El tercer elemento del capital intelectual es el Capital Relacional (CR), también llamado Capital Social (CS) que es el resultado de la inteligencia competitiva y social consubstanciada por el valor de las relaciones y acciones de la empresa compartidas con los agentes externos o sociales (IADE-CIC., 2003). Según Bontis (1999), es el conocimiento individual de los canales de mercado, clientes y proveedores, bien como el conocimiento del impacto de las asociaciones gubernamentales o industriales. Ejemplo de ello está en las relaciones con los empleados; el CI no sólo in- 


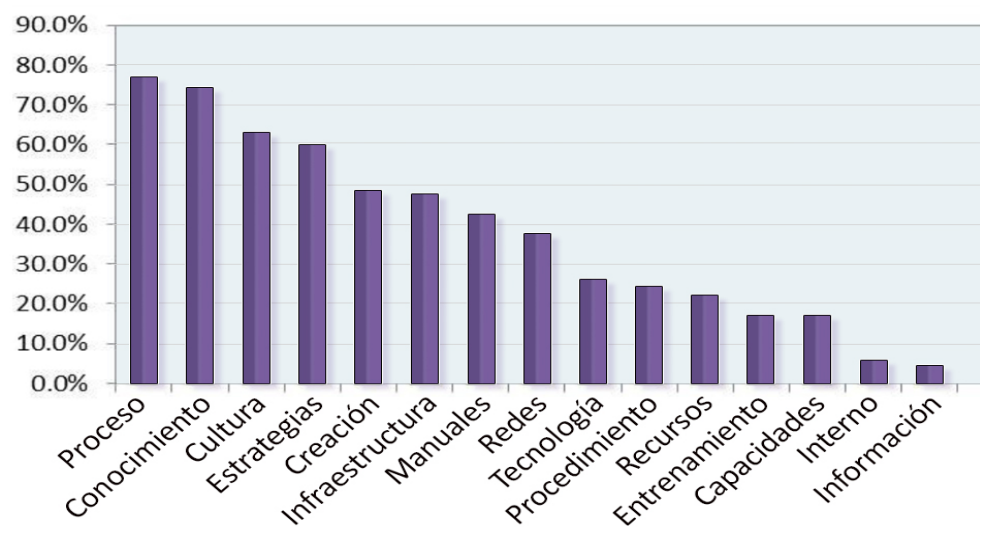

Figura 1. Quince palabras claves para definir CE Fuente: (Gogana et al., 2014)

cluye los contenidos de la mente o la mente de los empleados, sino que también incluye la estructura intangible y compleja entre ellos para realizar actividades y funciones organizacionales.

Según Trimurni \& Erlinac (2015) el Capital Social afecta significativamente la gobernanza a través del desarrollo del capital social, que es la capacidad de organizarse, construir una red de cooperación y participar en la sociedad. Una herramienta muy importante para la gestión empresarial es el cuadro de mando. Este instrumento, introducido por Kaplan y Norton (1992) hace que la tarea de evaluar el CI sea menos subjetiva.

\section{Gestión del conocimiento (GC)}

La GC es un nuevo y controvertido término que tiene muchas y diferentes definiciones. Fue introducido por primera vez en la Conferencia de Gestión de Europa en 1986. El Centro de Productividad y Calidad estadounidense define la GC como las estrategias y procesos de identificación, captura y aprovechamiento de los conocimientos (Atefeh et al, 1999, p. 172). Por otro lado, Knapp (1998) lo define como el arte de transformar la información y los activos intelectuales en un valor duradero para las organizaciones de clientes y su gente. Según Marulanda, Giraldo y López (2013) definen la GC como el proceso sistemático de buscar, organizar, filtrar y presentar información con el objetivo de hacerlo comprensible para las personas en un área específica de interés y a partir del mismo determinar qué se evalúa. Otros auto- res lo definen como un proceso que comprende crear, aplicar y transferir conocimientos en la organización, con el fin de conseguir ventaja competitiva de acuerdo a sus objetivos (Wiig, 1997; Alavi \& Leidner, 2001).

Darroch (2003) lo define como el proceso de crear, compartir, distribuir y utilizar el conocimiento en la organización. Otros investigadores han identificado diferentes procesos de GC: i) La creación, transferencia y aplicación (Spender, 1996), ii) Captura, transferencia y aplicación (De Long, 1997), iii) Identificación, captura, desarrollo, distribución, difusión, aplicación y almacenamiento (Probst et al, 2000).

Todos estos procesos, según la investigación realizada por Tarí \& García (2009), dan lugar a las dimensiones del conocimiento: a) Creación (Aprendizaje Organizativo), b). Almacenamiento y transferencia (Conocimiento Organizativo) y c) Aplicación y uso (Organización del Aprendizaje). De este modo, capturar, almacenar, compartir y distribuir conocimiento permiten innovar (Baptista et al., 2006).

Según Vidovic (2010) las ventajas de la GC sobre el Desempeño Organizativo, se ve en la tabla 1.

\section{Tabla 1}

Resultados de la gestión del conocimiento

\begin{tabular}{ll}
\hline $\begin{array}{l}\text { Grupo de } \\
\text { resultado }\end{array}$ & Resultados \\
\hline Rendimiento de & - Mejor toma de decisiones \\
los empleados & - Nuevas o mejores formas de trabajar \\
& - Comunicación mejorada \\
& - Habilidades mejoradas de los empleados \\
& - Mayor colaboración \\
& - Compartir mejores prácticas \\
\hline Desempeño de & Aumento de las ganancias \\
la Organización & - Reducción de costos \\
& - Aumento del empoderamiento de empleados \\
& - Mayor retenciónlatracción de los empleados \\
& - Rol de esfuerzos de Gestión Conocimiento \\
& - Aumento de las acciones \\
\hline Rendimiento & - Aumento del tamaño del mercado \\
del Mercado & Mayor mercado compartido \\
& - Calidad mejorada de producto o servicio \\
& - Creación de más valor para el cliente \\
& - Entrada a diferentes tipos de mercado \\
& Mejor manejo de Clientes
\end{tabular}

Elaboración propia.

Fuente: Anantatmula \& Kanungo, (2006, p.29) 
Asimismo, es relevante sustentar la importancia que tiene la GC para las organizaciones, según Tarí \& García (2013) la GC influye en los resultados operativos, financieros y de innovación en las organizaciones; según Arteche (2011), manifiesta que la GC tiene algunos retos que debe cumplir. Por ejemplo: cómo la organización puede transformarse en una organización inteligente; también refiere que su factor de éxito está en función de determinar cuál es el modelo adecuado. En esta adecuación es ideal apoyarse en un sistema de gestión de conocimiento (SGC) que permita unificar la estrategia de GC y la estrategia de proceso de la organización empresarial con el fin de alcanzar los objetivos organizacionales. Considerando que los SGC son:

Una clase de sistemas de información aplicados para gestionar conocimiento organizativo y desarrollados para soportar y mejorar procesos de creación, almacenamiento, recuperación, transferencia y aplicación del conocimiento" (Alavi \& Leidner, 2001, p. 107).

Por su parte Anantatmula \& Kanungo (2006) insisten en la importancia de la medición de la gestión del conocimiento y citan tres razones para medir el éxito de un sistema de gestión del conocimiento: 1) proporcionar una base para la valoración, 2) estimular enfoque de la administración en lo que es importante y 3) para justificar las inversiones.

Gobernanza y Gestión de la tecnologías de la información

Para dejar claros los términos que hacen referencia a Gobernanza, gobierno corporativo y gobierno de las TI, se describen a continuación algunas definiciones que especifican estos aspectos.

La Gobernanza Corporativa, según el Comité de Organizaciones Patrocinadoras de la Comisión de Normas (COSO, 2013) y la Organización para la Cooperación y el Desarrollo Económico (OCDE, 2004) hacen mención que es; la provisión de la estructura que permite determinar los objetivos de la organización y supervisar el rendimiento, a fin de asegurar que los objetivos sean cumplidos; según la ISO/IEC 38500: 2015, el Gobierno
Corporativo se centra en seis principios: responsabilidad, estrategia, adquisición, rendimiento, conformidad y comportamiento humano, y tres funciones que son evaluar, dirigir y monitorizar.

Gobernanza de las TIC ISO 38500 - COBIT / Val IT, manifiestan que es la especificación del marco de derecho a la toma de decisiones y a la alta responsabilidad para favorecer un comportamiento deseable en el uso de las TIC.

El gobierno corporativo, para Haji, \& Ghazali, (2013), es un sistema o una forma en que las empresas se controlan para ser esta responsable de sus grupos de interés, relacionando además con el CI con los atributos del gobierno corporativo.

Respecto al Gobierno de la TI para que este desempeñe un gobierno idóneo se basa en los principios de gobierno corporativo para la gestión y el uso de las TI para alcanzar los objetivos empresariales. Para TIC-CRUE (2016) es un sistema que permite dirigir y controlar la utilización de las TI actuales y futuras; a través de la dirección y evaluación de planes de uso de estas y a su vez sirven de soporte a la organización. Además, pretenden atender las demandas externas (de los clientes) en un horizonte temporal futuro (Peterson, 2003). Para Toomey (2009) el foco del Gobierno de la TI lleva directamente al modelo más básico de los negocios: Planifica, Construye y Ejecuta (véase área izquierda de la figura 2).
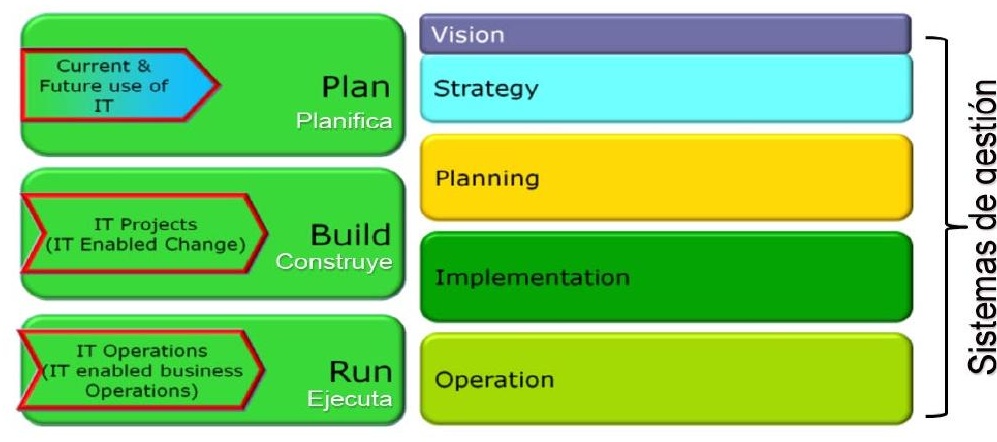

Figura 2. Principales sistemas de la gestión TI. Fuente (Toomey, 2009). 


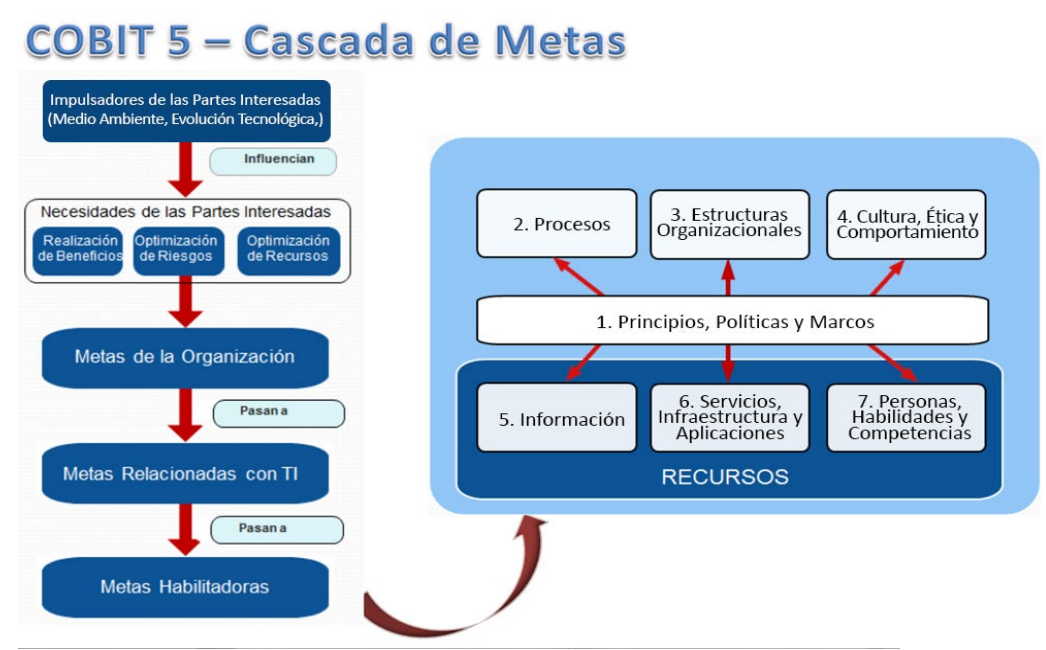

Figura 4. Cascada de metas desde Gobierno de TI hacia la gestión de TI. Fuente: (ISACA, 2014, Pág. 7)

Tabla 2

Revisión de la literatura de artículos

\begin{tabular}{|c|c|c|c|}
\hline Referencia & Nombre del artículo & Relación & Método/Aporte \\
\hline $\begin{array}{l}\text { Abd Rahman } \\
\text { et.al., } 2015\end{array}$ & $\begin{array}{l}\text { El nivel de conocimiento del Gobierno } \\
\text { Corporativo en los órganos federales } \\
\text { estatutarios en Malasia - } 2015\end{array}$ & $\begin{array}{l}\text { Importancia de la GC en el } \\
\text { consejo de administración }\end{array}$ & $\begin{array}{l}\text { Examina el nivel de conocimiento de la adminis- } \\
\text { tración sobre el concepto, principios y prácticas de } \\
\text { gobierno corporativo (encuestas). }\end{array}$ \\
\hline $\begin{array}{l}\text { Jameelah, } \\
\text { et.al.,2015 }\end{array}$ & $\begin{array}{l}\text { El efecto del capital intelectual sobre el } \\
\text { rendimiento de las organizaciones }\end{array}$ & $\begin{array}{l}\text { Efecto del } \mathrm{Cl} \text { en el rendi- } \\
\text { miento }\end{array}$ & $\begin{array}{l}\text { Usa } 6 \text { elementos del } \mathrm{Cl} \text { y analiza su aporte al rendi- } \\
\text { miento (otros estudios lo reagrupar y queda } 3 \text { ) }\end{array}$ \\
\hline $\begin{array}{l}\text { Sadalia \& } \\
\text { Nurbaity, } 2015\end{array}$ & $\begin{array}{l}\text { Análisis discriminante del capital inte- } \\
\text { lectual - Universidad de Medan }\end{array}$ & $\begin{array}{l}\text { El Gobierno Corporativo } \\
\text { tiene una gran influencia } \\
\text { sobre el } \mathrm{Cl}\end{array}$ & $\begin{array}{l}\text { Usa método análisis discriminante apoyado con } \\
\text { SPSS }\end{array}$ \\
\hline $\begin{array}{l}\text { Todericiu \& } \\
\text { Serban, } 2015\end{array}$ & $\begin{array}{l}\text { Capital Intelectual y su relación con las } \\
\text { Universidades }\end{array}$ & $\begin{array}{l}\text { Relación } \mathrm{Cl} \text { en las Univer- } \\
\text { sidades }\end{array}$ & $\begin{array}{l}\text { Trata de investigar el papel del } \mathrm{Cl} \text { en las organiza- } \\
\text { ciones de hoy en día modernos y, en particular, su } \\
\text { relevancia para las instituciones educativas. }\end{array}$ \\
\hline $\begin{array}{l}\text { Gogana, et.al., } \\
2014\end{array}$ & $\begin{array}{l}\text { Capital Estructural - un modelo de } \\
\text { medición propuesto }\end{array}$ & Capital Estructural & $\begin{array}{l}\text { Identifica los elementos de la CE que ayudan a } \\
\text { asegurar el éxito de la organización. }\end{array}$ \\
\hline $\begin{array}{l}\text { Mahfoudh \& } \\
\text { Izah., } 2012\end{array}$ & $\begin{array}{l}\text { Características de rendimiento del } \\
\text { capital intelectual y de la Junta de } \\
\text { Bancos del CCG }\end{array}$ & $\begin{array}{l}\mathrm{Cl} \text { y el rendimiento, mide } \\
\text { el rendimiento del } \mathrm{Cl} \text { a } \\
\text { través de método del valor } \\
\text { añadido del coeficiente } \\
\text { intelectual (VAIC) desarro- } \\
\text { llado por Pulic (1998) }\end{array}$ & $\begin{array}{l}\text { Examina la relación entre las características de la } \\
\text { mesa de directores (diversidad nivel de estudios, la } \\
\text { diversidad nacionalidad, tabla de enclavamiento, ta- } \\
\text { maño del mesa directiva y el número de consejeros } \\
\text { independientes) y el rendimiento }\end{array}$ \\
\hline Vidovic, 2010 & $\begin{array}{l}\text { Enlace entre la calidad de la GC y } \\
\text { el rendimiento financiero: Caso de } \\
\text { Croacia }\end{array}$ & $\begin{array}{l}\text { Elección de la GC y } \\
\text { rendimiento }\end{array}$ & $\begin{array}{l}\text { Usa ROS y ROA como medidas de rendimiento } \\
\text { organizacional }\end{array}$ \\
\hline $\begin{array}{l}\text { Sayyed, et. al., } \\
2011\end{array}$ & $\begin{array}{l}\text { Examinando el impacto de los facili- } \\
\text { tadores de la GC sobre los procesos } \\
\text { de GC }\end{array}$ & $\begin{array}{l}\text { Impacto de los facilitadores } \\
\text { de GC sobre los procesos } \\
\text { básicos }\end{array}$ & Encuentra una relación directa \\
\hline $\begin{array}{l}\text { Beinborn, et.al., } \\
2009\end{array}$ & $\begin{array}{l}\text { Propuesta de un modelo teórico para } \\
\text { Gobernanza de TI y alineamiento del } \\
\text { negocio con } \mathrm{TI}\end{array}$ & $\begin{array}{l}\text { Al explicar el alineamiento } \\
\text { se soporta sobre el } \mathrm{Cl}: \mathrm{CH} \text {, } \\
\mathrm{CR}, \mathrm{CS}\end{array}$ & $\begin{array}{l}\text { Elaborar un modelo que será explicado y empírica- } \\
\text { mente evaluado sobre el alineamiento }\end{array}$ \\
\hline
\end{tabular}

Elaboración propia. 
En la tabla 3 se relacionan los siete habilitadores y se asocian a la CI y a la GC. Se observa que todos los habilitadores están relacionados con la GC (columna 3) y sólo algunos están asociados al CI (columnas 4, 5 y 6), según el análisis realizado.

Tabla 4

Habilitadores y su relación con la GC y CI

\begin{tabular}{|c|c|c|c|c|c|}
\hline \multirow[b]{2}{*}{ Catalizador } & \multirow{2}{*}{$\begin{array}{l}\text { Interacción entre Gobier- } \\
\text { no y Gestión de la TI en } \\
\text { COBIT } 5\end{array}$} & \multirow[b]{2}{*}{$\mathrm{GC}$} & \multicolumn{3}{|l|}{$\mathrm{Cl}$} \\
\hline & & & $\mathrm{CH}$ & CE & $\mathrm{CR}$ \\
\hline $\begin{array}{l}\text { Principios, } \\
\text { Políticas y } \\
\text { Marcos }\end{array}$ & $\begin{array}{l}\text { Son los vínculos mediante } \\
\text { el cual las decisiones del } \\
\text { Gobierno (establecen orien- } \\
\text { taciones) son ejecutadas en } \\
\text { la gestión. }\end{array}$ & $\sqrt{ }$ & & $\sqrt{ }$ & + \\
\hline Procesos & $\begin{array}{l}\text { En los procesos catalizado- } \\
\text { res se distingue procesos } \\
\text { de Gobierno y de Gestión, } \\
\text { incluyes prácticas y activi- } \\
\text { dades para c/u, inclusive a } \\
\text { la matriz RACl. }\end{array}$ & $\sqrt{ }$ & & $\sqrt{ }$ & + \\
\hline $\begin{array}{l}\text { Estructuras } \\
\text { organizativas }\end{array}$ & $\begin{array}{l}\text { En cada empresa se } \\
\text { definen varias estructuras } \\
\text { de organizativas en función } \\
\text { de su composición y ámbito } \\
\text { de decisiones. }\end{array}$ & $\sqrt{ }$ & & $\sqrt{ }$ & + \\
\hline $\begin{array}{l}\text { Cultura Ética } \\
\text { y Comporta- } \\
\text { mientos }\end{array}$ & $\begin{array}{l}\text { El comportamiento es } \\
\text { un catalizador del buen } \\
\text { Gobierno y de Gestión, se } \\
\text { establece en el más alto } \\
\text { nivel. }\end{array}$ & $\sqrt{ }$ & $\sqrt{ }$ & $\sqrt{ }$ & + \\
\hline Información & $\begin{array}{l}\text { El modelo de procesos } \\
\text { describe las I/O de los dis- } \\
\text { tintos procesos, basados en } \\
\text { prácticas de otros procesos, } \\
\text { incluyendo la información } \\
\text { intercambiada entre los } \\
\text { procesos de Gobierno y de } \\
\text { Gestión. }\end{array}$ & $\sqrt{ }$ & $\sqrt{ }$ & $\sqrt{ }$ & + \\
\hline $\begin{array}{l}\text { Servicios, } \\
\text { Infraestructura } \\
\text { y Aplicaciones }\end{array}$ & $\begin{array}{l}\text { Se requieren servicios so- } \\
\text { portados por las aplicacio- } \\
\text { nes e infraestructura, para } \\
\text { proporcionar información } \\
\text { adecuada al órgano de } \\
\text { Gobierno, para Dirigir, } \\
\text { Evaluar y Monitorear. }\end{array}$ & $\sqrt{ }$ & & $\sqrt{ }$ & + \\
\hline $\begin{array}{l}\text { Personas, } \\
\text { habilidades y } \\
\text { Competencias }\end{array}$ & $\begin{array}{l}\text { Las actividades de Gobier- } \\
\text { no y de Gestión requieren } \\
\text { un conjunto de habilida- } \\
\text { des distintas. Estas son } \\
\text { requeridas para completar } \\
\text { exitosamente todas las } \\
\text { actividades y para tomar las } \\
\text { decisiones correctas, así } \\
\text { como para llevar a cabo las } \\
\text { acciones correctivas. }\end{array}$ & $\sqrt{ }$ & $\sqrt{ }$ & & + \\
\hline
\end{tabular}

Elaboración propia.
Según COBIT 5-marco (2013) en todo momento las organizaciones y sus ejecutivos están haciendo esfuerzos para:

- Crear y mantener información de calidad para apoyar las decisiones del negocio.

- Generar valor comercial a sus inversiones habilitadas por la TI; vale decir lograr metas estratégicas y mejoras al negocio mediante el uso eficaz e innovador de la TI.

- Lograr una excelencia operativa mediante la aplicación eficiente y fiable de la tecnología.

- Mantener y controlar el riesgo relacionado con TI a niveles aceptables.

- Optimizar el costo y los servicios de TI, entre otros.

Estas preocupaciones necesitan ser puestas en contexto y diferenciar los roles que competen. Para lograr valor para las partes interesadas se requiere un buen gobierno y una buena administración de los activos de TI y de la información.

COBIT 5 proporciona este marco integral que ayuda a las organizaciones a lograr sus metas y entregar valor mediante un gobierno y una administración efectiva de la TI, como se puede observar en la figura 5.

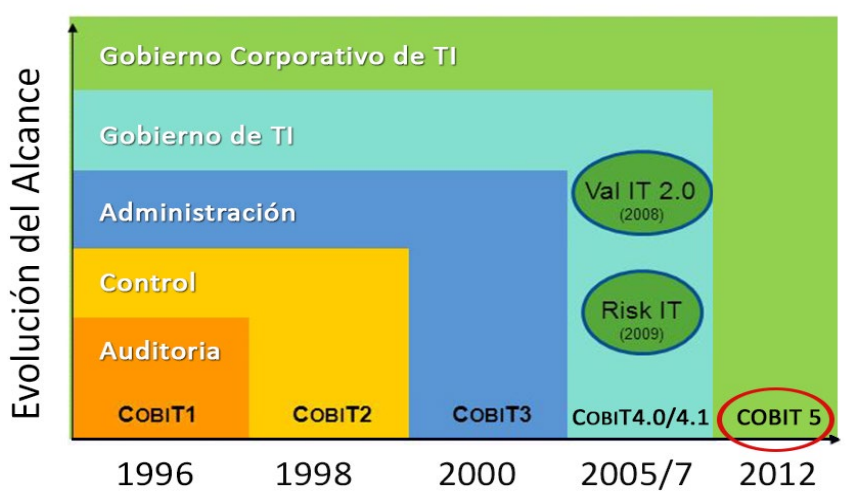

Figura 5. Marco empresarial de ISACA.

Fuente: (CiGRAS, 2014).

COBIT 5 une los cinco principios que permiten a la organización construir un marco efectivo de Gobierno y Administración basado en una serie holística de siete habilitadores, que optimizan la inversión en tecnología e información, así como su uso en beneficio de las partes interesadas. Los 
principios y habilitadores de COBIT 5 son genéricos y útiles para las organizaciones de cualquier tamaño bien sean comerciales, sin fines de lucro o en el sector público. Al basarse en cinco principios y siete habilitadores, COBIT 5 utiliza prácticas de gobierno y gestión para describir las acciones que son ejemplo de mejores prácticas de su aplicación.

Los cinco principios de COBIT 5:

1. Satisfacer las necesidades de las partes interesadas.

2. Cubrir la compañía de forma integral.

3. Aplicar un solo marco integrado.

4. Habilitar un enfoque holístico.

5. Separar el gobierno de la administración.

Los siete habilitadores se esquematizan en la figura 6 , así como su relación.

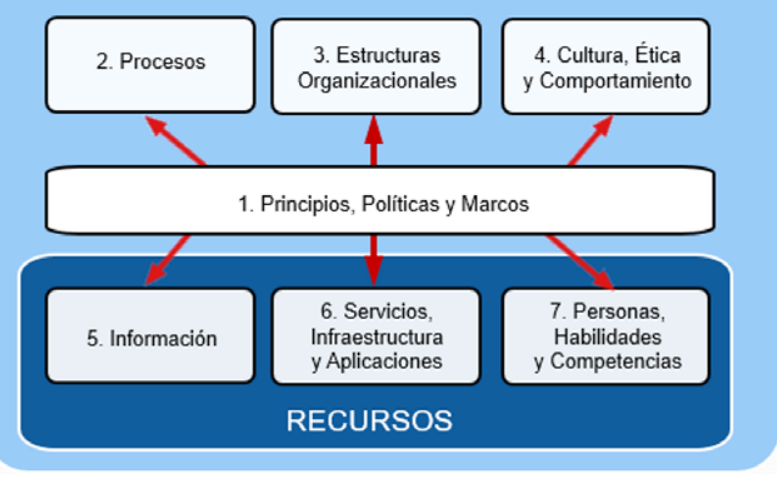

Figura 6. COBIT 5 Habilitadores de empresa. Fuente: (COBIT 5, 2012, Pág. 27)

\section{HABILITADORES Y SU ANÁLISIS RELACIO-}

\section{NADO A LA GC Y CI}

De acuerdo a la propuesta se detalla a continuación cada uno de los siete habilitadores en uso de COBIT 5.

\section{Principios, Políticas y Marcos (1)}

"Son los vehículos para traducir el comportamiento deseado en guías prácticas para la gestión día a día" (COBIT 5, 2012). Estos son determinados por el órgano de dirección más alto, se sus- tenta en la parte normativa (definida en su plan estratégico y las buenas prácticas según manuales de referencia), las políticas y la cultura organizacional que corresponden al CE y la ejecución es realizada por las personas $(\mathrm{CH})$; pero esa interacción humana se denomina $\mathrm{CR}$, que se da según las mejores prácticas usando las herramientas pertinentes, etc. (CE).

\section{Procesos (2)}

"Describen un conjunto organizado de prácticas y actividades para alcanzar ciertos objetivos y producir un conjunto de resultados que soporten las metas generales relacionadas con la TI" (COBIT 5, 2012).

Entendiéndose que hay procesos a nivel de gobierno, así como a nivel de gestión cada uno con sus entradas y salidas propias. Se sustentan en los manuales respectivos de cada institución donde se consignan la descripción de los procesos automatizados o no. Esta documentación corresponde al CE y es resultado de la generación de conocimiento y registro en su fase de combinación propiamente dicha (Nonaka \& Takeuchi, 1995).

\section{Estructuras Organizacionales (3)}

"Son las entidades de toma de decisiones clave en una organización” (COBIT 5, 2012). Corresponde a una adecuación dinámica de las organizaciones a efectos de lograr sus objetivos estratégicos, estas estructuras deben estar definidas en el Manual de Organización y Funciones (MOF) donde se describen puestos, dependencias jerárquicas etc., corresponde al CE.

El modelo propuesto por Tallon, et al. (2013) asocia al GTI usando artefactos de Gobierno de la Información; el enfoque dominante de la literatura de GTI ha sido cómo las empresas gobiernan los artefactos físicos de las TI (hardware, software y redes). El objetivo del modelo es ampliar la teoría del GTI mediante el descubrimiento de las estructuras y prácticas utilizadas para gobernar artefactos de información. Se incorpora y se observaron cómo las prácticas de Gobierno de la Información pueden desbloquear el valor de los crecientes datos dentro de las organizaciones. Se 
precisa que los activos de información están dentro del CE.

\section{Cultura, Ética y Comportamiento (4)}

"De los individuos y de la empresa, son muy a menudo subestimados como factor de éxito en las actividades de gobierno y gestión" (COBIT 5, 2012).

Se puede hablar de ética organizacional, determinada por los valores que la empresa ha definido y ética individual relaciona a cada individuo $(\mathrm{CH})$. Incluso, se podría hablar de ética de equipo. La cultura y la ética conllevan a un comportamiento deseado de las personas el cual debe tener como horizonte los objetivos institucionales. La cultura se sustenta en el CE y la ética y el comportamiento están asociados al $\mathrm{CH}$.

\section{La Información (5)}

"Impregna toda la organización e incluye toda la información producida y utilizada por la empresa. La información es necesaria para mantener la organización funcionando y bien gobernada, pero a nivel operativo, la información es muy a menudo el producto clave de la empresa en sí misma” (COBIT 5, 2012). Y esta se encuentra presente en todo el ambiente de cualquier organización; por otro lado, la información es la fuente donde se genera el conocimiento al ser incorporado al individuo (internalización o socialización del proceso de generación de conocimiento). La información está registrada en diferentes medios, por ende, corresponde a CE; también el conocimiento está registrado en la mente de las personas; pero cuando se expresa es información, motivo por el cual corresponde al $\mathrm{CH}$ y en el caso que se haya registrado en algún sistema basado en conocimiento estaría en el contexto del CE. También la información es el insumo que facilita la interacción entre agentes permitiendo obtener el CR.

\section{Servicios, Infraestructura y Aplicaciones (6)}

"Incluyen la infraestructura, la tecnología y las aplicaciones que proporcionan a la empresa servicios y tecnología de procesamiento de la información” (COBIT 5, 2012).
La información integrada y almacenada en algún soporte tecnológico (infraestructura) relacionada con alguna aplicación para lograr algún objetivo institucional se traduce hacia ese entorno como un servicio permitiendo interactuar, comunicarse etc., para lograr el objetivo previsto.

En aras de mejorar la confianza de los clientes hay un experimento de Huang et al. (2011) usando las TI aplicando COBIT; en este trabajo los autores desarrollan y prueban un nuevo factor en su modelo, la confianza en el comercio electrónico (e-comercio): La banca por Internet. El control interno de la banca por Internet es muy consistente con los altos niveles de los factores de confianza como la seguridad, la privacidad y otros temas de riesgo. Sin embargo, este tipo de asociación aún no ha sido ampliamente reconocido como un modelo de confianza desde el punto de vista del consumidor de comercio electrónico. Este estudio trata de crear nuevos factores en la GTI de COBIT donde los autores consideran el sello de confianza virtual como Sello de confianza del Gobierno de la TI de COBIT, se propone ampliar los vínculos de la empresa con un nuevo servicio (e-comercio) y lo importante es darle la confianza y seguridad, el cual pasa por crear una cultura en ambas entidades, manifestándose el $\mathrm{CR}$, mientras que la infraestructura, cultura y servicio corresponden al CE.

\section{Personas, Habilidades y Competencias (7).}

"Están relacionadas con las personas y son necesarias para poder completar de manera satisfactoria todas las actividades, para la correcta toma de decisiones y de acciones correctivas" (COBIT 5, 2012).

Este habilitador corresponde al $\mathrm{CH}$, el conocimiento reside en las personas que tienen las habilidades, capacidades y competencias para realizar el cambio que requiere la institución y eso se manifiesta mediante el comportamiento pragmático en la ejecución de las directivas y realización de las buenas prácticas con ética para poder realizar ese cambio organizacional que le permita ser cada vez más competitivo y sostenible a la organización.

Un factor determinante es la cultura organizacio- 
nal, según Robbin (2004) la cultura es un conjunto de valores, creencias, normas, procedimientos y significados compartidos por los miembros de la organización.

De la revisión de la literatura se observa que para la evaluación de la GTI en las organizaciones ya se está considerando a la GC como el motor para ese cambio esperado, se puede confirmar en el enfoque de Bin-Abbas, H. \& Bakry, S. (2014) que esta es una arista del pentágono del método STOPE (Strategy, Technology, Organization, People and Environment); para la integración de los diferentes dominios están usando la Gestión del Conocimiento (personas con $64.9 \%$ y estas son parte integral del capital humano $(\mathrm{CH})$ ).

Este método permite la integración de diferentes dominios donde los autores Bin-Abbas \& Bakry (2014) lo integran con los principios de la GC como un valor añadido y las fases de Six Sigma como un proceso de mejora cíclica. Asimismo, sostiene que el problema con el uso y aplicación de las recomendaciones de GTI disponibles, referencias de evaluación etc., son diversas y en algunos casos largas y complicadas. También considera que estas recomendaciones no proporcionan la suficiente atención a los principios de GC. Por tanto, surge la necesidad de unificar y simplificar la evaluación de gobernabilidad, lográndose mejorar dichas evaluaciones teniendo en cuenta los principios de GC. El enfoque STOPE, que desarrollan para superar estos inconvenientes lo agrupan en cinco dominios en los que se ha prestado especial atención al factor humano en la gestión de TI a través de dos consideraciones principales:

1. GC se asocia directamente con la conducta humana; y

2. La consideración de un dominio independiente para las personas entre los cinco dominios principales del ámbito de GTI considerado (figura 7).

Con esta simplicidad y definida las competencias, las personas pueden fácilmente desarrollar las habilidades para el logro de los objetivos institucionales, siguiendo las guías y marcos establecidos.

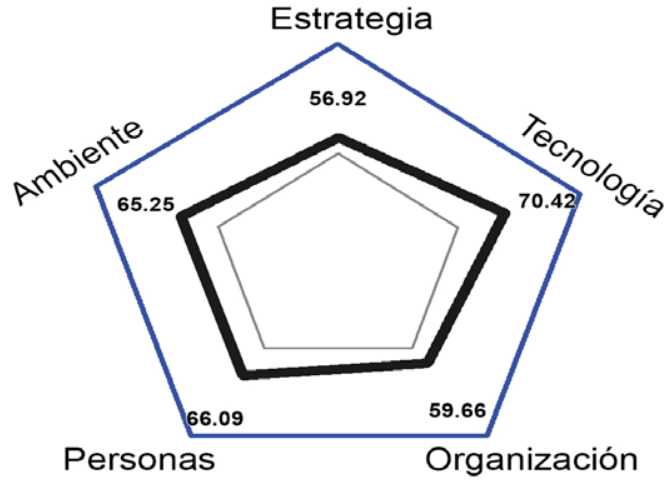

Figura 7. Porcentaje de Rendimiento del Gobierno de la TI aplicando STOPE

Fuente: (Bin-Abbas, H. \& Bakry, S., 2014).

\section{Conclusiones}

El capital intelectual es tan importante como la inversión de capital para las empresas.

La Gestión del CI debe ser parte del cuerpo de conocimiento y uso del Gobierno Corporativo y consecuentemente en la Gestión de la TI. Se sugiere tener cuidado en los conceptos de gobierno corporativo, gobernanza de TI, GTI a fin de tener una visión clara y precisa en cuanto a sus alcances y aportes.

El gobierno de la organización se refiere al marco de responsabilidad global que coordina todas las actividades de gestión respecto a todos los stakeholders (partes interesadas), mientras que el gobierno corporativo corresponde principalmente a la junta o consejo de gobierno, el equipo de gestión ejecutiva y los accionistas. El gobierno de las TI, por su parte, se centra en el uso de la tecnología para satisfacer los objetivos de la organización fijados por la dirección. Por ello, el gobierno corporativo incluye aspectos del gobierno de las TI, ya que, sin una gestión eficaz de las TI los encargados de las responsabilidades corporativas no podrían desempeñarse de forma efectiva (Fink et ál., 2006).

Los resultados del SGC se producen y se observan en el largo plazo, este escenario podría ser la razón por la cual los gerentes desconocen el funcionamiento de la GC en sus organizaciones, consecuentemente no hay el apoyo ni el compromiso respectivo o en todo caso es tardío. 
Capital Intelectual, Gestión del Conocimiento en la Interacción Gobierno y Gestión de la Tecnologías de la Información desde Perspectiva COBIT 5

Se recomienda que el responsable del área de TI/ SI sea parte integrante del comité de gobierno de TI para asegurar un alineamiento más efectivo.

De la interacción entre el Gobierno y la Gestión de la TI, partiendo de ISO/IEC 38500: 2015 y el COBIT 5 (en función del cuarto principio y siete habilitadores) se identificó la fuerte relación de la GC y CI y cómo estos son agentes naturales del cambio.

El buen uso de la GC por parte de una organización la hace más competitiva, el uso de la GC en la organización soportado por la tecnología la convierte en una organización inteligente. Esta organización inteligente tiene como objetivo su desarrollo y sostenibilidad y quién asegura estos logros son los marcos y las buenas prácticas determinadas por COBIT 5, entre otras.

Uno de los problemas de los marcos de gobierno sea ISO/IEC 38500: 2015 o el COBIT 5, inclusive Calder-Moir (2013) afirma que no precisan la gestión de los activos intangibles como así tampoco las bondades que puede ofrecer la GC para facilitar su ejecución de sus directivas.

Se Considera que se deben realizar más investigaciones y profundizar el tema a fin que se entienda y se revaloricen las potencialidades y aportes del CI y la GC.

Como trabajos futuros se recomienda hacer una revisión más exhaustiva en cuanto a la participación como agentes de cambio de la GC y CI en cualquier modelo o marco de Gestión de TI.

\section{REFERENCIAS BIBLIOGRÁFICAS}

Abd Rahman, H., Mustaffa, M., Zubaidah, Z. \& Roslani, E. (2015). The Level of Knowledge of Corporate Governance in Federal Statutory Bodies in Malaysia, 7th International conference on financial criminology 2015 13-14 April 2015, Wadham College, Oxford, United Kingdom.

Akpinar, A. T. \& Akdemir, A. (1999). Intellectual capital, in third European Conference, 332-340. Recuperado de: http://joanes.opf.slu.cz/vvr/akce/turecko/pdf/Akpinar.pdf

Alavi, M. \& Leidner, D. (2001). Review. Knowledge management and knowledge management systems: conceptual foundations and research issues, MIS Quarterly, 25(1), 107136. Recuperado de: https://doi.org/10.2307/3250961

Amrizah, K. \& Rashidah, A. (2013). Intellectual Capital Profiles: Empirical Evidence of Malaysian Companies Kamaluddin \& Rahman. International Review of Business Research Papers, 9(6), 83-101. Recuperado de: http://irbrp. $\mathrm{com} /$ static/documents/November/2013/6

Anantatmula, V. \& Kanungo, S. (2006). Structuring the underlying relations among the knowledge management outcomes. Journal of Knowledge Management, 10(4), 25-42. Recuperado de: https://doi. org/10.1108/13673270610679345

Arteche, R. (2011). Retos y alternativas de la gestión del conocimiento (GC) como propuesta para la colaboración en organizaciones inteligentes. Educar, 47(1), 121-138.

Atefeh, S., McCamble, L., Moorchead, C. \& Gitters, S. (1999). Knowledge management: the new challenge for the 21 century. Journal of Knowledge Management, 3(3), 172-179. Recuperado de: https://doi. org/10.1108/13673279910288572

Baptista, M., Annansingh, F., Eaglestone, B. \& Wakefield, R. (2006). Knowledge management issues in knowledge-intensive SMEs. Journal of Documentation, 62 (1), 101-119. Recuperado de: https://doi. org/10.1108/00220410610642075

Barney, J. B. (1991). Firm Resource and Sustained Competitive Advantage. Journal of management, 17(1), 99-120. Recuperado de: https://doi.org/10.1177/014920639101700108

Beimborn, D., Franke, J., \& Weitzel, T. (2009). Proposing a theoretical model for IT governance an IT business alignment. 42nd Hawaii International Conference on System Sciences, Big Island, HI, USA.

Bin-Abbas, H. \& Bakry, S. (2014). Assessment of IT governance in organizations: A simple integrated approach. Computers in Human Behavior 32, 261-267. Recuperado de: https://doi.org/10.1016/j.chb.2013.12.019

Bontis, N. (1999). Managing Organizational Knowledge by Diagnosing Intellectual Capital: Framing andadvancing the state of the field. International Journal of Technology Management, 18 (5,6,7,8), 433-463. Recuperado de: https://doi. org/10.1504/IJTM.1999.002780

Bontis, N., Keow,W. \& Richardson, S. (2000). Intellectual capital and business performance in Malaysian industries. Journal of Intellectual Capital, 1 (1), 85 - 100. Recuperado de: https://doi.org/10.1108/14691930010324188

Calder-Moir, (2013). It Governance Framework. The IT Governance \& ISO38500. Recuperado de: http://www. itgovernance.co.uk/download/Calder-Moir-v2.pdf

Chen, M., Cheng, S. \& Hwang, Y. (2005). An empirical investigation of the relationship between intellectual capital and firms' market value and financial performance. Journal of Intellectual Capital, 6(2), 159-176. Recuperado de: https://doi.org/10.1108/14691930510592771

Chen, Y., Wang, Y., Nevo, S., Jin, J. F., Wang, L., \& Chow, 
W. S. (2014). IT capability and organizational performance: the roles of business process agility and environmental factors. European Journal of Information Systems, 23, 326342. Recuperado de: https://doi.org/10.1057/ejis.2013.4

COBIT 5, (2012). Framework "COBIT" 5: Enabling Processes” ISACA. Rolling Meadows, USA.

CiGRAS (2014) Como aporta COBIT 5 y gobernanza de TI a la gobernanza empresarial. V Congreso Internacional sobre Gobierno, Riesgos, Auditoria y Seguridad de la Información. ISACA. Capitulo Montevideo. Recuperado de: https://m.isaca.org/chapters8/Montevideo/cigras/Documents/CIGRAS2014-COBIT\%20y\%20Gobernanza\%20 de\%20TI.pdf

COBIT 5-marco, (2013). Un Marco de Negocio para el Gobierno y la Gestión de las TI de la Empresa. ISACA-Framework. Recuperado de: https://articulosit.files.wordpress. com/2013/07/cobit5-framework-spanish.pdf

COSO (2013). Committee of Sponsoring Organizations of the Treadway Commission. Control Interno - Marco Integrado. Resumen Ejecutivo. Traducido al español. Instituto de Auditores Internos de España. Recuperado de: http:// doc.contraloria.gob.pe/Control-Interno/Normativa_Asociada/coso_2013-resumen-ejecutivo.pdf

Darroch, J. (2003). Developing a measure of knowledge management behaviors and practices. Journal of knowledge management, 7(5), 41-54. Recuperado de: https://doi. org/10.1108/13673270310505377

De Long, D. (1997). Building the knowledge-based organization: How culture drives knowledge behaviors. Center for Bussines Innovation, Ernst \& Young: LLP.

Fink, D., Huegle, T. y Dortschy, M. A (2006). Model of Information Security Governance for E-Business. Idea Group Publishing. Recuperado de: https://doi.org/10.4018/978-159140-911-3.ch001

Fitz-Enz, J. (2009). The ROI of Human Capital, American Management Association. N. Y., USA.

Gogana, L., Durana. D. \& Draghici, A. (2014). Structural capital - A proposed measurement model, 2nd Global Conference on Business economics, management and tourist, 30-31 October, 2014, Prague, Czech Republic.

Haji, A. \& Ghazali, N. (2013). A longitudinal examination of intellectual capital disclosures and corporate governance attributes in Malaysia. Asian Review of Accounting, 21 (1), 27 - 52. Recuperado de: https://doi. org/10.1108/13217341311316931

Huang, S.-M., Shen, W.-C., Yen, D. \& Chou, L.-Y. (2011). IT governance: Objectives and assurances in internet banking. Advances in Accounting, incorporating Advances in International Accounting 27, 406-414. Recuperado de: https://doi.org/10.1016/j.adiac.2011.08.001

IADE-CIC (2003). Modelo Intellectus: medición y gestión del capital intelectual. Documentos Intelectos, Madrid.

ISACA (2014). COBIT 5. Niveles de Capacidad. Desafío de Formalización de procesos costo y beneficios. Cigras,
Capítulo Montevideo. Crowe Horwath. Recuperado de: http://www.isaca.org/chapters8/Montevideo/cigras/Documents/Cigras_2014_COBIT\%205\%20-\%20Niveles\%20 de\%20Capacidad.pdf

Jameelah. M., Osmanb. I., Musa., S (2015). Effect of Intellectual Capital on Organizational Performance, 2nd Global Conference on Business and Social Science-2015, GCBSS-2015, 17-18 September 2015, Bali, Indonesia.

Kaplan R. \& Norton P., (1992). The Balanced Scorecard -Measures that Drive Performance, Harvard Business Review. PMid:10119714

Knapp, E.M. (1998). Knowledge Management. Business and Economic Review, 44(4), 3-7. Recuperado de: https:// goo.gl/zCW6hp

Mahfoudh. A. \& Izah., N. (2012). "Intellectual Capital Performance and Board Characteristics of GCC Banks". Procedia Economics and Finance, 2, 219-226. Recuperado de: https://doi.org/10.1016/S2212-5671(12)00082-2

Marco-Empresarial (2012). Un marco empresarial completo. Recuperado de: www.isaca.org/cobit

Marulanda, C. E., Giraldo, J. A., y López, M. (2013). Evaluación de la gestión del conocimiento en las organizaciones de la red de tecnologías de información y comunicaciones del eje cafetero en Colombia, Inf. tecnol., 24 (4), 23-24. Recuperado de: https://doi.org/10.4067/S071807642013000400012

Nonaka, I. \& Takeuchi, H. (1995). The knowledge-creating company: How Japanese companies create the dynamics of innovation. New York: Oxford University Press.

OCDE, (2004). Principios de Gobierno Corporativo de la OCDE (Organización para la cooperación y el desarrollo económico). (Traduccción). Recuperado de: Palao, M. (2010). Reflexión sobre el Estado del Arte del Buen Gobierno TIC. Bogotá: ISACA. Recuperado de: https://doi. org/10.1787/9788485482726-es

Peterson, R. (2003). Integration Strategies and Tactics for Information Technology Governance. Strategies for Information Technology Governance. Hershey, PA: Idea Group Publishing.

Pham, T. (2005). An empirical study of knowledge transfer within Vietnam's information technology companies. Recuperado de: https://pdfs.semanticscholar.org/e08d/da9cb0559133a04f7339c1dc7cab43a903d4.pdf

Probst, G., Raub, S. \& Romhardt, K. (2000). Managing Knowledge: Building Blocks for Success, New York: John Wiley \& Sons.

Robbin, S. P. (2004). Organizational behavior (11th ed). Upper Saddle River, NJ: Prentice-Hall.

Rodríguez, J., Aguilar, M. y Raudales, N. (2017). Una mirada breve al software para la Gestión del Conocimiento. RIDTEC, 13(1).

Sadalia, I. y Nurbaity, A. (2015). Discriminant Analysis of Intellectual Capital Model of State University in Medan, 2nd Global Conference on Business and Social Science-2015, 
Capital Intelectual, Gestión del Conocimiento en la Interacción Gobierno y Gestión de la Tecnologías de la Información desde Perspectiva COBIT 5

GCBSS-2015, 17-18 September 2015, Bali, Indonesia

Sayyed, M. A., Sayyed. M. Z. \& Sayyed. M. R, (2011). Examining the Impact of KM Enablers on Knowledge Management Processes. Procedia Computer Science, 3, 1211-1223. Recuperado de: https://doi.org/10.1016/j. procs.2010.12.196

Spender, J. C. (1996). Organizational Knowledge, learning and memory: three concepts in search of a theory. Journal of organizational change management 9(1), 63- 78. Recuperado de: https://doi.org/10.1108/09534819610156813

Steward, T. A. (1998). La nueva riqueza de las organizaciones: el capital intelectual, Chile: Granica.

Tallon, P., Ramírez, R. \& Short, J. (2013). The Information Artifact in IT Governance: Toward a Theory of Information Governance. Journal of Management Information Systems 30(3), 41-177. Recuperado de: https://doi.org/10.2753/MIS0742-1222300306

Tarí, J. y García, M. (2009). Dimensiones en la gestión del conocimiento y de la gestión de la calidad: Una revisión de la literatura. Investigaciones Europeas de Dirección y Economía de la Empresa, 15 (3), 135-148. Recuperado de: https://doi.org/10.1016/S1135-2523(12)60105-1

Tarí, J. y García, M. (2013). ¿Puede la gestión del conocimiento influir en los resultados empresariales? Cuadernos de Gestión, 13 (1) 151-176. Universidad del País Vasco/Euskal Herriko Unibertsitatea Vizcaya, España

Teece, D. J., (2000). Strategies for managing knowledge assets: the role of firmstructure and industrial context. Long Range Plann., 33, 35-54. Recuperado de:

https://doi.org/10.1016/S0024-6301(99)00117-X

TIC-CRUE (2016). Gobierno de las tecnologías de la información en universidades. Conferencia de rectores de las universidades españolas. Recuperado de: http://tic.crue.org/ wp-content/uploads/2016/04/FolletoGobiernoTI.pdf

Todericiu, R. \& Serban. A (2015). Intellectual Capital and its Relationship with Universities, 22nd International Economic Conference - IECS 2015. "Economic Prospects in the Context of Growing Global and Regional Interdependencies".

Toomey, M. (2009). A Framework for Governance and Management of IT. The infonomics Letter, mayo.

Trimurni. L. \& Erlinac, S. (2015). The Effect of Social Capital on Governance and Sustainable Livelihood of Coastal City Community Medan, 2nd Global Conference on Business and Social Science-2015, GCBSS-2015, 17-18 September 2015, Bali, Indonesia.

Vidovic, M. (2010). The link between the quality of knowledge management and financial performance - The case of Croatia.

Villegas, E., Hernández, M. y Salazar, B. (2015). La medición del capital intelectual y su impacto en el rendimiento financiero en empresas del sector industrial en México. Contaduría y Administración, 62, 184-206. Recuperado de: https://doi.org/10.1016/j.cya.2016.10.002
Wiig, K. M. (1997). Knowledge Management: An Introduction and Perspective. Journal of Knowledge Management, 1(1), 6-14. Recuperado de: https://doi. org/10.1108/13673279710800682 